\title{
Heterodyne pump probe measurements of nonlinear dynamics in an indium phosphide photonic crystal cavity
}

Heuck, Mikkel; Combrié, S.; Lehoucq, G.; Malaguti, S.; Bellanca, G.; Trillo, S.; Kristensen, Philip Trøst; Mørk, Jesper; Reithmaier, J. P.; de Rossi, A.

Published in:

Applied Physics Letters

Link to article, DOI:

$10.1063 / 1.4828355$

Publication date:

2013

Document Version

Publisher's PDF, also known as Version of record

Link back to DTU Orbit

Citation (APA):

Heuck, M., Combrié, S., Lehoucq, G., Malaguti, S., Bellanca, G., Trillo, S., Kristensen, P. T., Mørk, J.,

Reithmaier, J. P., \& de Rossi, A. (2013). Heterodyne pump probe measurements of nonlinear dynamics in an indium phosphide photonic crystal cavity. Applied Physics Letters, 103(18), [181120].

https://doi.org/10.1063/1.4828355

\section{General rights}

Copyright and moral rights for the publications made accessible in the public portal are retained by the authors and/or other copyright owners and it is a condition of accessing publications that users recognise and abide by the legal requirements associated with these rights.

- Users may download and print one copy of any publication from the public portal for the purpose of private study or research.

- You may not further distribute the material or use it for any profit-making activity or commercial gain

- You may freely distribute the URL identifying the publication in the public portal 


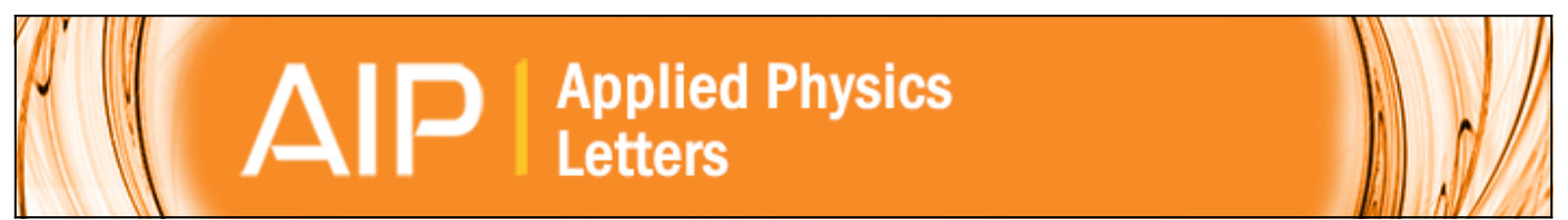

Heterodyne pump probe measurements of nonlinear dynamics in an indium phosphide photonic crystal cavity

M. Heuck, S. Combrié, G. Lehoucq, S. Malaguti, G. Bellanca, S. Trillo, P. T. Kristensen, J. Mørk, J. P. Reithmaier , and A. de Rossi

Citation: Applied Physics Letters 103, 181120 (2013); doi: 10.1063/1.4828355

View online: http://dx.doi.org/10.1063/1.4828355

View Table of Contents: http://scitation.aip.org/content/aip/journal/apl/103/18?ver=pdfcov

Published by the AIP Publishing

\section{Over 700 papers \& presentations on \\ multiphysics simulation vew now}

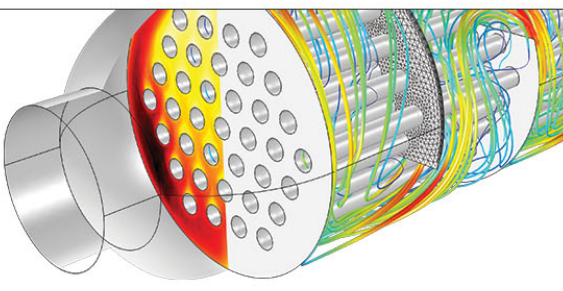




\title{
Heterodyne pump probe measurements of nonlinear dynamics in an indium phosphide photonic crystal cavity
}

\author{
M. Heuck, ${ }^{1}$ S. Combrié, ${ }^{2}$ G. Lehoucq,${ }^{2}$ S. Malaguti, ${ }^{3}$ G. Bellanca, ${ }^{3}$ S. Trillo, ${ }^{3}$ \\ P. T. Kristensen, ${ }^{1}$ J. Mørk, ${ }^{1, a)}$ J. P. Reithmaier, ${ }^{4}$ and A. de Rossi ${ }^{2, b)}$ \\ ${ }^{1}$ DTU Fotonik, Technical University of Denmark, DK-2800 Kgs. Lyngby, Denmark \\ ${ }^{2}$ Thales Research and Technology, Palaiseau Cedex FR 91767, France \\ ${ }^{3}$ Department of Engineering, University of Ferrara, Via Saragat 1, IT 44122 Ferrara, Italy \\ ${ }^{4}$ Institute of Nanostructure Technologies and Analytics, University of Kassel, 34132 Kassel, Germany
}

(Received 23 September 2013; accepted 15 October 2013; published online 1 November 2013)

\begin{abstract}
Using a sensitive two-color heterodyne pump-probe technique, we investigate the carrier dynamics of an InP photonic crystal nanocavity. The heterodyne technique provides unambiguous results for all wavelength configurations, including the degenerate case, which cannot be investigated with the widely used homodyne technique. A model based on coupled mode theory including two carrier distributions is introduced to account for the relaxation dynamics, which is assumed to be governed by both diffusion and recombination. (C) 2013 AIP Publishing LLC.
\end{abstract}

[http://dx.doi.org/10.1063/1.4828355]

It is well-known from waveguide propagation studies that caution must be exercised when interpreting the results of homodyne pump probe measurements. ${ }^{1}$ The reason is that the photo current from the detector will contain a contribution originating both from the modulation of the material properties caused by the strong pump and experienced by the weak probe and vice versa, unless the pump pulse can be blocked before the detector using spectral or polarization filtering. When characterizing structures intended for alloptical switching, it is the change in transmission of the probe due to the presence of the pump, which is the relevant figure of merit. ${ }^{2}$ Thus, the contribution stemming from the change of the material caused by the probe and experienced by the pump may be considered an artifact of the measurement technique in this case.

Previously, experimental investigations of the cavity time dynamics in semiconductor photonic crystal (PhC) structures have used a homodyne measurement technique. In this work, we use a two-color heterodyne pump-probe technique, ${ }^{3}$ which enables a clear separation of the contributions mentioned above. Furthermore, we perform independent measurements both in the time- and spectral-domain to extract additional data to be used in a comparison with modeling results. Our experimental results show that the recovery of carriers after optical excitation occurs in stages governed by different decay times. This is in line with previous work using InGaAsP. ${ }^{4}$ In InP, the ambipolar diffusion length exceeds $1 \mu \mathrm{m}$ easily, which is larger than the average distance the carriers have to travel in order to reach the surface of the $\mathrm{PhC}$ or to escape the region of high optical field. Indeed, very strong surface effects are considered to lie behind the fast dynamics of GaAs PhC cavities. ${ }^{5}$ In other materials, such as InGaAsP, ${ }^{4}$ silicon, ${ }^{6}$ and InP, considered here, the diffusion plays a crucial role, as demonstrated through the solution of the carrier diffusion equation. It was suggested in Refs. 4 and 6 that the decay rate of the initial

\footnotetext{
${ }^{a)}$ Electronic mail: jesm@fotonik.dtu.dk

b)Electronic mail: alfredo.derossi@thalesgroup.com
}

fast change of the carrier distribution depends on the size of the cavity and it was estimated to be 3.5 ps (Ref. 4) for an $\mathrm{H} 0$ cavity, which is much faster than the typical recombination time in InP. However, in Ref. 4 the carrier dynamics was not connected to the observed gate recovery time in a quantitative manner. Here, a model of the carrier dynamics is combined with temporal coupled mode theory ${ }^{7}$ (CMT) and an extensive comparison with the measurements is carried out. The considered device is shown in Fig. 1 and it consists of a single mode $\mathrm{H} 0$ cavity with a small modal volume, based on the design of Zhang and Qiu, ${ }^{8}$ and one input and output waveguide as in Refs. 4 and 5. The lower right inset in Fig. 1 shows the details of the structure with the parameters: Lattice spacing $a=470 \mathrm{~nm}$, hole radius $0.22 a$, cavity shifts $\delta x=0.15 a$ (green circles), $\delta y=0.07 a$ (orange circles), and radius of the first row of holes adjacent to the waveguide $r_{1}=0.3 a$ (red circles). The self-standing membrane structure is obtained by etching a $250 \mathrm{~nm}$ thick layer of InP grown by molecular beam epitaxy. The superimposed magnetic induction field $\left|H_{z}\right|^{2}$ of the cavity mode ${ }^{9}$ in Fig. 1 is obtained from 3D finite difference time domain (FDTD) simulations, as the resonant field resulting from an initial short input pulse.

Our model of the carrier recovery is an attempt at including the diffusion effect by simple rate equations, and it is inspired by finite element simulations of the diffusion equation, see Refs. 4 and 6. Initially, the generated carriers assume a distribution given by the optical energy density for linear absorption and the square of the optical energy distribution in the case of two photon absorption (TPA). Diffusion then causes the carriers to spread out, and after some time the overlap with the optical energy distribution reaches an approximately constant and much smaller value. At this stage the recovery is dominated by carrier recombination. To describe this complicated process using only ordinary differential equations, we introduce ambipolar carrier density amplitudes $N_{1}$ and $N_{2}$ for the initial and slow stage carrier distributions. The rate of exchange of carriers between the distributions is assumed to be proportional to the density 


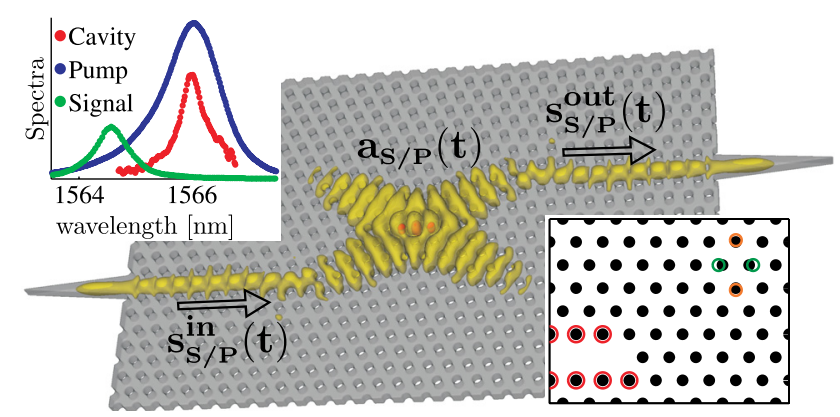

FIG. 1. Contour surfaces of the calculated mode profile (yellow and red correspond to $2 \%$ and $50 \%$ of $\max \left\{\left|H_{z}\right|^{2}\right\}$, respectively) of the $\mathrm{H} 0$ cavity structure used in the experiments (the waveguides are shorter than in the real device). The variables of the coupled mode model are also indicated. The insets show the measured input spectra and linear transmission spectrum of the cavity as well as a zoom-in of the cavity region.

difference $\Delta N=N_{1}-N_{2}$, since the particle current is proportional to the density gradient in the diffusion equation. ${ }^{10}$ The time evolution of the carrier amplitudes is then given by

$$
\begin{gathered}
\dot{N}_{1}(t)=-\gamma_{\text {diff }} \Delta N(t)-\gamma_{\mathrm{nr}} N_{1}(t)+\frac{K_{\mathrm{TPA}}^{\mathrm{P}}\left|a_{\mathrm{P}}(t)\right|^{4}}{\hbar \omega_{\mathrm{C}} V_{1}}, \\
\dot{N}_{2}(t)=\gamma_{\text {diff }} \frac{V_{1}}{V_{2}} \Delta N(t)-\gamma_{\mathrm{nr}} N_{2}(t) .
\end{gathered}
$$

The generation of carriers in $\mathrm{InP}$ around $1550 \mathrm{~nm}$ is caused by TPA and is proportional to the square of the cavity field energy $\left|a_{\mathrm{P}}\right|^{2}$ through the coefficient $K_{\mathrm{TPA}}^{\mathrm{P}}$. The decay rates of the two stages are $\gamma_{\text {diff }}$ and $\gamma_{\mathrm{nr}}$ and the parameters $V_{1}$ and $V_{2}$ are normalization volumes associated with $N_{1}$ and $N_{2}$, such that $V_{1} N_{1}$ and $V_{2} N_{2}$ are the total number of carriers in each distribution. The ratio $V_{1} / V_{2}$ is a measure of the rate at which the distribution with amplitude $N_{2}$ saturates, and thus, it determines whether the fast or slow time constant will dominate. The nonlinear dynamics of nanocavities is usually understood within the framework of $\mathrm{CMT}^{11,12}$ where it is assumed that all nonlinear effects can be included as perturbative corrections to the complex eigenfrequency of the cavity $\tilde{\omega}_{\mathrm{C}}=\omega_{\mathrm{C}}-\mathrm{i} \gamma_{\mathrm{C}} \cdot{ }^{13}$ Following this approach, Eq. (1) is coupled to the dynamical CMT equations for the cavity fields by the relations

$$
\begin{gathered}
\dot{a}_{\mathrm{S} / \mathrm{P}}=\left[-\mathrm{i} \delta_{\mathrm{S} / \mathrm{P}}-\gamma_{\mathrm{C}}-\mathrm{i} \delta_{\mathrm{NL}}^{\mathrm{S} / \mathrm{P}}\right] a_{\mathrm{S} / \mathrm{P}}+\sqrt{\gamma} s_{\mathrm{S} / \mathrm{P}}^{\mathrm{in}}, \\
\delta_{\mathrm{NL}}^{\mathrm{S} / \mathrm{P}}=\left(K_{\mathrm{FCD}}-\mathrm{i} K_{\mathrm{FCA}}\right) N_{1}-\left(K_{\mathrm{Kerr}}^{\mathrm{S} / \mathrm{P}}+\mathrm{i} K_{\mathrm{TPA}}^{\mathrm{S} / \mathrm{P}}\right)\left|a_{\mathrm{P}}\right|^{2} .
\end{gathered}
$$

The cavity (waveguide) fields $a_{\mathrm{S} / \mathrm{P}}\left(s_{\mathrm{S} / \mathrm{P}}^{\text {in }}\right)$ are normalized so $\left|a_{\mathrm{S} / \mathrm{P}}\right|^{2}$ is the cavity energy and $\left|s_{\mathrm{S} / \mathrm{P}}^{\text {in }}\right|^{2}$ is the power in the waveguide, where $\mathrm{S}$ and $\mathrm{P}$ represent the signal and pump, respectively. The outputs are related to the cavity fields by $s_{\mathrm{S} / \mathrm{P}}^{\text {out }}=\sqrt{\gamma} a_{\mathrm{S} / \mathrm{P}}$, and $\delta_{\mathrm{S} / \mathrm{P}}=\omega_{\mathrm{C}}-\omega_{\mathrm{S} / \mathrm{P}}$ is the detuning of the incoming signal or pump from the cavity resonance. $\gamma / 2$ is the decay rate into the waveguides, $\tau_{1}$ is the delay in arrival between the pump and the signal, and $\delta_{\mathrm{NL}}^{\mathrm{S} / \mathrm{P}}$ are the nonlinear resonance shifts.

The effective coefficients for the Kerr effect $K_{\mathrm{Kerr}}^{\mathrm{S} / \mathrm{P}}$ and TPA $K_{\mathrm{TPA}}^{\mathrm{S} / \mathrm{P}}$ are connected to the real and imaginary parts of the third-order susceptibility tensor $\chi^{(3)}$ via an effective complex energy, ${ }^{14}$ which is calculated from perturbation theory for the cavity mode. The coefficients related to free carrier effects are calculated using the theory in Ref. 13 with a perturbation $\Delta \epsilon_{r}(\mathbf{r}, t)=f_{N_{1}}(\mathbf{r}) N_{1}(t) \partial \epsilon / \partial N_{1}$, where $f_{N_{1}}(\mathbf{r})$ $=V_{1}\left|\tilde{f}_{\mathrm{C}}(\mathbf{r})\right|^{4} / \int\left|\tilde{f}_{\mathrm{C}}(\mathbf{r})\right|^{4} d V$ inside the crystal and $f_{N_{1}}=0$ in the air holes, while $\tilde{f}_{\mathrm{C}}(\mathbf{r})$ is the electric field distribution of the cavity mode. Note that only $N_{1}$ is assumed to interact with the field in Eq. (2b) due to the much smaller overlap between $f_{N_{2}}$ and $\left|\tilde{f}_{\mathrm{C}}(\mathbf{r})\right|^{4}$. Equation (2) is based on the assumption that the cavity field can be separated into two distinct parts, $a_{\mathrm{P}}$ and $a_{\mathrm{S}}$, even though the pump and signal are interacting with the same cavity resonance. The model neglects wave mixing effects between the signal and pump, except for the instantaneous Kerr and TPA contributions, which are accounted for by setting $K_{\mathrm{Kerr}}^{\mathrm{S}}=2 K_{\mathrm{Kerr}}^{\mathrm{P}}$ and $K_{\mathrm{TPA}}^{\mathrm{S}}=2 K_{\mathrm{TPA}}^{\mathrm{P}}$. The coherent term $a_{\mathrm{S}}^{*} a_{\mathrm{P}}^{2}$ may be neglected due to its different microwave frequency, which causes it to be filtered out by the lock-in amplifier, and second order terms in $a_{\mathrm{S}}$ are neglected since the signal is generally much weaker than the pump.

In the experiments, the input optical pulses are obtained by filtering the spectrally large output of a 100fs modelocked fiber laser, operating at a repetition rate $l_{\text {rep }}$ of 36.5 MHz, with narrowband filters with widths of $1.2 \mathrm{~nm}$ and $0.7 \mathrm{~nm}$ for the pump and signal, respectively. The filters have nearly Lorentzian spectral transmission, so the input fields have a triangular shape in the time domain, which we model by exponentials with a rise time of $0.8 \mathrm{ps}$ and fall times of $2.2 \mathrm{ps}$ and $3.6 \mathrm{ps}$ for the pump and signal. The energy arriving at the cavity $U_{\mathrm{P}}^{\text {in }}$ is related to the average power measured outside the sample $\bar{P}_{\mathrm{P}}$ by $U_{\mathrm{P}}^{\text {in }}=\bar{P}_{\mathrm{P}} T_{\text {coup }} / l_{\text {rep }}$, where $T_{\text {coup }}$ includes both coupling- and waveguide loss. The set-up for the heterodyne experiment is sketched in Fig. 2. After the band-pass filter, the signal is further split and two acoustooptic modulators (AOMs) shift the frequency of the signal and the reference by 80 and $82 \mathrm{MHz}$, respectively. At the output, the signal interferes with the reference at a beam splitter, and the detected signal is passed to a lock-in amplifier, which measures the component of the photo current oscillating at the difference frequency between the AOMs. The lock-in signal is given by

$$
L\left(\tau_{1}\right)=\int\left(\int_{\text {pulse }}\left|s_{\mathrm{S}}^{\text {in }}\left(t-\tau_{2}\right) s_{\mathrm{S}}^{\text {out } *}\left(t-\tau_{1}\right)\right| d t\right)^{2} d \tau_{2}
$$

The inner integration in Eq. (3) is taken over the duration of a single pulse, while the outer integration is done by postprocessing of the lock-in data to remove the dependence on the reference delay $\tau_{2}$. The time-domain experiment is

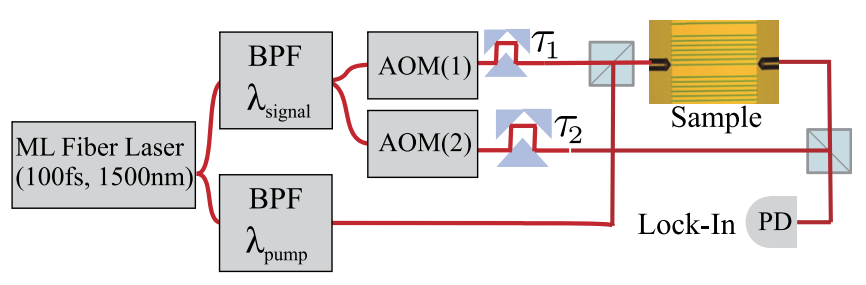

FIG. 2. Schematic illustration of the experimental setup used to perform the two-color heterodyne pump-probe measurements. ML: mode locked, BPF: band-pass filter, AOM: acousto-optic modulator, PD: photo detector. 
performed in both the switch-on configuration, where the signal is blue-detuned from the resonance, and in the switchoff configuration, where the signal is on resonance. The results are shown in Figs. 3(a) and 3(c), respectively. As expected, the signal output becomes larger (smaller) close to zero delay, when the signal is blue-detuned (on-resonance), because the cavity resonance is blue-shifted due to free carrier dispersion (FCD). From the tail of the lock-in signal, the carrier recovery is clearly observed to happen fast initially, and then develop into a slower stage at larger delays.

In the spectral-domain experiment, the signal is blocked and only the pump is coupled into the sample and its spectral broadening is measured using an optical spectrum analyzer (Arnitsu, $\Delta \lambda=50 \mathrm{pm}$ ). The pump wavelength coincides with the cold cavity resonance $\lambda_{\mathrm{C}}$ and Fig. 3(e) shows the output spectra at different power levels. The output spectra are observed to broaden towards shorter wavelengths with increasing pump energy, which is again consistent with a decrease in the refractive index caused by FCD. Even though the transmission blue-shifts, a significant part of the output
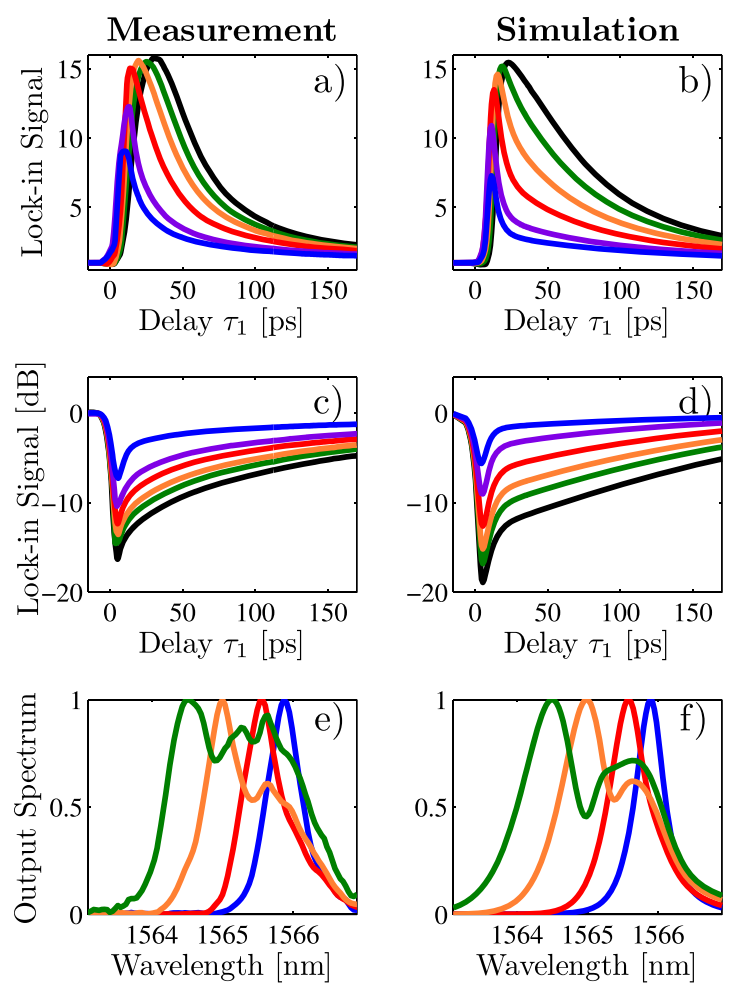

FIG. 3. Measurement (left column) and simulation (right column) results. The upper and center panels are time-domain experiments showing the lock-in signal as a function of the pump-signal delay $\tau_{1}$ for different pump powers. The curves are normalized to 1 at large negative delays. The bottom panel shows spectral-domain experiments, where the output spectrum of the pump was measured at different power levels. (a) The power levels are $\bar{P}_{\mathrm{P}}=15,20,30,40,50,60 \mu \mathrm{W}$ corresponding to the colors blue to black, the signal wavelength is $\lambda_{\mathrm{S}}=1564.5 \mathrm{~nm}$ and the pump wavelength is $\lambda_{\mathrm{P}}=1566.2 \mathrm{~nm}$. (b) The coupling is $T_{\text {coup }}=0.37$ and the cold cavity resonance is $\lambda_{\mathrm{C}}=1566.5 \mathrm{~nm}$. (c) The power levels are $\bar{P}_{\mathrm{P}}=7,12,20$, $30,40,60 \mu \mathrm{W}$ corresponding to the colors blue to black, and the wavelengths are $\lambda_{\mathrm{S}}=\lambda_{\mathrm{P}}=1566.2 \mathrm{~nm}$. (d) The coupling used is $T_{\text {coup }}=0.24$ and $\lambda_{\mathrm{C}}=1566.1 \mathrm{~nm}$. (e) The power levels are $\bar{P}_{\mathrm{P}}=10,20,40,60 \mu \mathrm{W}$ corresponding to the colors blue to green, $\lambda_{\mathrm{P}}=1566.2 \mathrm{~nm}$, and the spectra are normalized to a maximum of one. (f) The coupling is $T_{\text {coup }}=0.14, \lambda_{\mathrm{C}}=1566.1 \mathrm{~nm}$, and the curves are normalized as in (e). The quality factor used in all simulations is $Q=\omega_{\mathrm{C}} / 2 \gamma_{\mathrm{C}}=3200$, corresponding to an energy decay time of $2.7 \mathrm{ps}$, and the remaining parameters are listed in Table I. spectrum is located near $\lambda_{\mathrm{C}}$ because the resonance shift happens dynamically during the passage of the pulse, which allows a part of the pump energy centered at $\lambda_{\mathrm{C}}$ to be transmitted. This gives rise to the double peak feature observed in Fig. 3(e).

Figs. 3(b), 3(d), and 3(f) show simulation results using the model described by Eqs. (1) and (2). The qualitative agreement with the experimental data is good, but we note that the quantitative agreement requires the coupling $T_{\text {coup }}$ and cold cavity resonance $\lambda_{\mathrm{C}}$ to be adjusted differently for each measurement series corresponding to Figs. 3(b), 3(d), and 3(f). We believe this was necessary due to: (a) a drift of the positioning stages during the measurements, leading to changes in the coupled power and (b) irreversible changes of the sample caused by photo-induced oxidation, ${ }^{15}$ when operating at power levels that provide large enough switching contrasts to be relevant from an application point of view. The change in resonance frequency is in the range of a few hundreds of pm, which is large enough that it must be taken into account in the modeling, in order to compare with the different measurement series. The photo-induced changes were also observed to steepen the transmission roll-off in the measured spectra, which deteriorates the Lorentzian approximation assumed by the model. (c) Failure of the simple carrier rate equation model to fully and quantitatively capture all the diffusion dynamics.

By including the adjustments of $T_{\text {coup }}$ and $\lambda_{\mathrm{C}}$, the model is able to account for the saturation in the peak of the lock-in signal as well as its shift to larger delays with increasing pump power, which is observed in Fig. 3(a). We believe this originates from a combination of a large TPA and the nonlinear resonance shift exceeding the detuning at large power levels.

It is important to note that $L\left(\tau_{1}\right)$ from Eq. (3) is not directly related to the output energy of the signal, and hence, the switching contrast cannot be determined from Figs. 3(a)-3(d). However, the output energy is easily calculated from the model, and the contrast corresponding to the peak of the black curve in Fig. 3(b) is 10.7.

The parameters found by fitting to the measured data are listed in Table I. The coefficients in Eqs. (1) and (2) have been converted into parameters more commonly found in the literature. For comparison, we found the following values: $\beta_{\mathrm{TPA}}=14.6 \mathrm{~cm} / \mathrm{GW},{ }^{17} \beta_{\mathrm{TPA}}=66.6 \mathrm{~cm} / \mathrm{GW}$ (Ref. 18) (found by extrapolating the reported values of $\operatorname{Im}\left\{\chi_{\text {xxxx }}^{(3)}\right\}$ to a wavelength of $1566 \mathrm{~nm}$ ), and $\sigma_{\mathrm{abs}}=7.2 \times 10^{-17} \mathrm{~cm}^{2} .{ }^{17}$ The value of $\partial \epsilon_{\mathrm{FCD}} / \partial N_{1}$ in Table I is found from the Drude model in Ref. 19 using an effective mass $m_{e}=0.075 m_{0}$ and a mobility $\mu_{e}=5400 \mathrm{~cm}^{2} /(\mathrm{Vs})$ of InP. The parameter values we found by fitting to the experimental data are seen to be in reasonable agreement with the literature, thus providing confidence in the model.

In conclusion, we have investigated the free carrier dynamics in an InP PhC all-optical resonant switch, based on free carrier dispersion. The heterodyne measurement technique allowed us to perform an extensive comparison with a CMT model including carrier dynamics for both the switchon and switch-off configuration. The model seems to work well in accounting for the qualitative features of both timeand spectral domain experiments. Finally, we note that the 
TABLE I. Parameters used in Figs. 3(b), 3(d), and 3(f). Here, we state the commonly used parameters, and their relations to the coefficients in Eqs. (1) and (2) are given by $\beta_{\mathrm{TPA}}=3 \omega \operatorname{Im}\left\{\chi^{(3)}\right\} / 2 c^{2} \epsilon_{0} \epsilon_{\mathrm{r}}$ and $n_{2}=4 \operatorname{Re}\left\{\chi^{(3)}\right\} /$ $4 c \epsilon_{0} \epsilon_{\mathrm{r}}$, see Ref. 16. Comparing to Ref. 17 we find $\sigma_{\mathrm{abs}}=2 K_{\mathrm{FCA}} n_{0} / c$, where $c$ is the speed of light in vacuum, $\epsilon_{0}$ is the vacuum permittivity, and $n_{0}=\sqrt{\epsilon_{\mathrm{r}}}$. For the structure used here, the following relations are found using the results in Refs. 13 and 14 with a field distribution $\tilde{f}_{\mathrm{C}}$ calculated from FDTD: $K_{\text {Kerr }}^{\mathrm{P}}=18.7 \gamma_{\mathrm{C}} n_{0}^{3} /\left(\epsilon_{0} \lambda_{\mathrm{C}}^{3}\right) \times \operatorname{Re}\left\{\chi^{(3)}\right\}, \quad K_{\mathrm{TPA}}^{\mathrm{P}}=21.5 \gamma_{\mathrm{C}} n_{0}^{3} /\left(\epsilon_{0} \lambda_{\mathrm{C}}^{3}\right) \times \operatorname{Im}\left\{\chi^{(3)}\right\}$, $K_{\mathrm{FCD}}=-467 \gamma_{\mathrm{C}} \times \partial \epsilon_{\mathrm{FCD}} / \partial N_{1}$, and $K_{\mathrm{FCA}}=15.9 \gamma_{\mathrm{C}} \times \partial \epsilon_{\mathrm{FCA}} / \partial N_{1}$.

\begin{tabular}{ll}
\hline \hline$\partial \epsilon_{\mathrm{FCD}} / \partial N_{1}=-2.9 \times 10^{-26} \mathrm{~m}^{3}$ & $V_{1} / V_{2}=0.61$ \\
$\sigma_{\mathrm{abs}}=3.1 \times 10^{-17} \mathrm{~cm}^{2}$ & $1 / \gamma_{\mathrm{diff}}=8 \mathrm{ps}$ \\
$\beta_{\mathrm{TPA}}=81 \mathrm{~cm} / \mathrm{GW}$ & $1 / \gamma_{\mathrm{nr}}=175 \mathrm{ps}$ \\
$n_{2}=2.7 \times 10^{-17} \mathrm{~m}^{2} / \mathrm{W}$ & $\gamma / \gamma_{\mathrm{C}}=0.98$ \\
$V_{1}=1.5\left(\lambda_{\mathrm{C}} / n_{0}\right)^{3}$ & $\epsilon_{\mathrm{r}}=10.01$ \\
\hline \hline
\end{tabular}

homodyne and heterodyne techniques cannot be utilized in data transmission applications, which calls for multi-mode structures enabling the separation of the signal and pump. ${ }^{20}$

This work was supported by the EU FP7 project "Copernicus," the Villum Kann Rasmussen Center of Excellence NATEC, and the Danish council for independent research (FTP 10-093651). The InP material was grown in the framework of the project "GOSPEL." We thank Stephane Xavier for help in the fabrication of the sample and Yuriy Elesin for providing the FDTD code.
${ }^{1}$ A. Mecozzi and J. Mørk, J. Opt. Soc. Am. B 13, 2437 (1996).

${ }^{2}$ M. Heuck, P. T. Kristensen, and J. Mørk, Opt. Express 19, 18410 (2011).

${ }^{3}$ T. Katayama and H. Kawaguchi, IEEE Photonics Technol. Lett. 17, 1244 (2005).

${ }^{4}$ K. Nozaki, T. Tanabe, A. Shinya, S. Matsuo, T. Sato, H. Taniyama, and M. Notomi, Nature Photon. 4, 477 (2010).

${ }^{5}$ C. Husko, A. De Rossi, S. Combrié, Q. V. Tran, F. Raineri, and C. W. Wong, Appl. Phys. Lett. 94, 021111 (2009).

${ }^{6}$ T. Tanabe, H. Taniyama, and M. Notomi, J. Lightwave Technol. 26, 1396 (2008).

${ }^{7}$ C. Manolatou, M. J. Khan, S. Fan, P. R. Villeneuve, H. A. Haus, and J. D. Joannopoulos, IEEE J. Quantum Electron. 35, 1322 (1999).

${ }^{8}$ Z. Zhang and M. Qiu, Opt. Express 12, 3988 (2004).

${ }^{9}$ P. T. Kristensen, C. Van Vlack, and S. Hughes, Opt. Lett. 37, 1649 (2012).

${ }^{10}$ S. Elliott, The Physics and Chemistry of Solids, 3rd ed. (John Wiley \& Sons, Inc., 2005).

${ }^{11}$ B. Maes, P. Bienstman, and R. Baets, J. Opt. Soc. Am. B 22, 1778 (2005).

${ }^{12}$ Y. Dumeige, C. Arnaud, and P. Féron, Opt. Commun. 250, 376 (2005).

${ }^{13}$ H. M. Lai, P. T. Leung, K. Young, P. W. Barber, and S. C. Hill, Phys. Rev. A 41, 5187 (1990).

${ }^{14}$ M. Heuck, P. T. Kristensen, and J. Mørk, in Proceedings of Conference on Lasers and Electro-Optics, US, 2012 (IEEE, 2012), Vol. 2, pp. 1-3.

${ }^{15}$ F. Intonti, N. Caselli, S. Vignolini, F. Riboli, S. Kumar, A. Rastelli, O. G. Schmidt, M. Francardi, A. Gerardino, L. Balet, L. H. Li, A. Fiore, and M. Gurioli, Appl. Phys. Lett. 100, 033116 (2012).

${ }^{16}$ R. W. Boyd, Nonlinear Optics, 3rd ed. (Academic Press, London, 2008).

${ }^{17}$ L. P. Gonzalez, J. M. Murray, S. Krishnamurthy, and S. Guha, Opt. Express 17, 8741 (2009).

${ }^{18}$ T. Matsusue, H. Bando, S. Fujita, and Y. Takayama, Phys. Status Solidi C 8, 387 (2011).

${ }^{19}$ B. R. Bennett, R. A. Soref, and J. A. Del Alamo, IEEE J. Quantum Electron. 26, 113-122 (1990).

${ }^{20}$ Y. Yu, M. Heuck, S. Ek, N. Kuznetsova, K. Yvind, and J. Mørk, Appl. Phys. Lett. 101, 251113 (2012). 